\section{Information retrieval from two rapidly consecutive stimuli: A new analysis*}

\author{
GEORGE SPERLING \\ Bell Telephone Laboratories, Murray Hill, New Jersey 07974
}

Data from an experiment in which Ss reported letters from two consecutive stimuli are analyzed by a nonmetric multiple regression analysis that permits the evaluation of nonlinear-e.g., quantal-hypotheses. The data are shown to contradict the hypothesis that total stimulus duration is the critical factor in response accuracy and the hypothesis of a quantal time period. Instead, they support the theory of visual persistence and continuous processing (Sperling, 1963): namely, visibility of a stimulus persists for a few 10ths of a second after a stimulus has been turned off; the effect of a second stimulus exposure coming quickly after the first is to terminate visibility of the first stimulus and to substitute for it visibility of the second; information is retrieved at a rapid rate from whichever stimulus is visible.

Greenberg, Helfer, and Mayzner (1968), in an article henceforth called the source paper, recently reported on the ability of two $S s$ to extract information from consecutive briefly exposed stimuli. This article reanalyzes the data of the source paper by a new method and comes to somewhat different conclusions. Both the method of analysis and the new conclusions are of theoretical interest for the study of information processing.

\section{SUMMARY OF THE SOURCE PAPER \\ Procedure}

Ss view two consecutive briefly exposed stimuli, separated by a brief time interval. Each stimulus contains some letters, and the Ss' task is to write all the letters of both stimuli. Ss are scored correct only when they correctly identify all letters of both stimuli. Five conditions are studied that differ only in the type of stimulus material. The materials are: (1) single letter (each stimulus), (2) three random letters, (3) five random letters, (4) three-letter word, (5) five-letter word. The two members of each presentation pair contain different samples of letters taken from the same class of stimulus material. Testing conditions include four different exposure durations from 5 to $50 \mathrm{msec}$ (both stimuli were exposed for the same duration) and seven different interstimulus intervals, from 1 to $125 \mathrm{msec}$. The total number of different procedures is $5 \times 4 \times 7=140 .^{1}$

\section{Results}

Generally, response accuracy is observed to improve as exposure duration and/or interstimulus interval increases. This is true for all five classes of stimuli.

*Dr. J. D. Carroll contributed many helpful suggestions and, with Miss Jih-Jih Chang, performed the nonmetric multiple regression analysis. The editor, Dr. I. Pollack, contributed usetul suggestions for revision.
To analyze the data further, the source paper computes the total stimulus duration [duration of the first flash (T1) plus interstimulus interval (ISI) plus duration of the second flash (T2)] and relates this sum $(T 1+$ ISI $+T 2)$ to accuracy. As T1 equals $\mathrm{T} 2$ in all the experimental conditions, the total stimulus duration is simply $2 \mathrm{~T}+\mathrm{ISI}$. The source paper claims that a remarkable breakpoint occurs when the total stimulus duration is $50 \mathrm{msec}$ : there is poor performance below this duration and good performance above it. ${ }^{2}$

Because different stimuli having the same total stimulus duration occasionally give rise to different levels of performance, the source paper concludes that factors other than simply $2 \mathrm{~T}+$ ISI also contribute to response accuracy.

\section{SOURCE PAPER HYPOTHESIS}

\section{"Total Stimulus Duration" (2T + ISI)} Hy pothesis

From the source paper's listing of results in terms of $2 \mathrm{~T}+\mathrm{ISI}$, and from its subsequent interpretation of particular results as deviations from predictions based on $2 \mathrm{~T}+\mathrm{ISI}$, the implication might be drawn that $2 \mathrm{~T}+\mathrm{ISI}$ is the most appropriate description of the data. The first hypothesis to be evaluated, therefore, is that $2 T+$ ISI gives the best description of the data.

The underlying mechanism that might cause the total stimulus duration to be a critical variable is not described, but a suggestion is found in the source paper's unfortuante terminology: it consistently refers to the total stimulus duration $(2 \mathrm{I}+\mathrm{ISI})$ as the "total processing time." 3 In terms of mechanism, the first hypothesis may be restated as "total processing time equals total stimulus duration," where processing refers to mental processing and where response accuracy is assumed 10 be monotonically related to processing time.
Discontinuity (Quantal) Hypothesis

A second aspect of the data is the "large ... discontinuous break in percent correct detections between $51 \mathrm{msec}$ and $60 \mathrm{msec}$ " (of 2T+ISI), which the source paper believes may be the most important feature of the data. ${ }^{4}$ This large discontinuity suggests a psychological quantal time unit of $50 \mathrm{msec}$. On the other hand, the source paper correctly cites other data which do "not seem to fit easily into a 'moment' value model of $50 \mathrm{msec}$ duration."

There is a main and a subordinate hypothesis: (1) $2 \mathrm{~T}+\mathrm{ISI}$ is the appropriate description of the stimulus (i.e., accuracy is a monotonic function of $2 \mathrm{~T}+\mathrm{ISI}$ ); (2) there is a discontinuity in the data at $50 \mathrm{msec}$. The author has outlined elsewhere (Sperling, 1969) a quantal model that could predict the appropriateness of $2 \mathrm{~T}+$ ISI and a discontinuity in the derivative at $50 \mathrm{msec}$, but the model need not be described here because the subsequent analysis completely disconfirms the hypotheses. For ease of denotation the first hypothesis will be called the "total stimulus duration hypothesis," and the general class of discontinuity hypotheses (of which "discontinuity in 2T + ISI at $50 \mathrm{msec}^{\prime \prime}$ is a member) will be called "quantal hypotheses."

\section{THE VISUAL PERSISTENCE AND CONTINUOUS PROCESSING HYPOTHESIS}

Basic Assumptions (Sperling, 1963, 1967)

(1) Visibility of a brief visual stimulus usually persists for about $200 \mathrm{msec}$ or longer, depending on the extent to which stimulus conditions tend to produce afterimages. (2) When a second stimulus occurs before the persistence of the first has expired, the second stimulus becomes visible itself, thereby terminating visibility of the first. 5 (3) Letters can be retrieved (for later recall) from a visible stimulus at a maximum rate of about one letter per 10 to $15 \mathrm{msec}$ for the first three letters and at slower rates for the next few letters up to the limit of the memory span.

In the persistence theory, exposure duration perse is not a critical variable when the exposures are sufficiently intense to be legible. To predict the source paper's results from the persistence theory, we examine the time available to process each stimulus.

(1) Processing of the second stimulus is not interrupted by any third stimulus; visual persistence of even the shortest-duration second stimulus usually would suffice for correct identification of its elements.

(2) Processing of the first stimulus is interrupted by onset of the second. Therefore, the main factor to limit 
accuracy is the limited time available for processing the first stimulus. The time available for processing the first stimulus is simply the exposure duration of the first plus the interstimulus interval, that is, $\mathrm{T}+\mathrm{ISI}$.

\section{Hypothesis}

Accuracy of report is related monotonically and continuously to $\mathrm{T}+\mathrm{ISI}$.

\section{TEST OF THE HYPOTHESES}

To evaluate the main hypotheses, we may ask which hypothesis gives a better account of the data. Although it would be desirable to compare predictions of accuracy of report on each stimulus, first and second, data are given only for the overall combined accuracy. Therefore, to test the hypotheses, we ask whether response accuracy, the dependent variable, is better predicted by $2 \mathrm{~T}+$ ISI (source paper) or by $\mathrm{T}+\mathrm{ISI}$ (Sperling). To formulate a better question, we assume that response accuracy $y$ is a monotonic function of $\alpha \mathrm{T}+\beta \mathrm{ISI}$,

$$
\begin{aligned}
& y=M(\alpha \mathrm{T}+\beta \mathrm{ISI}) \text { or } \\
& \mathrm{M}^{-1}(\mathrm{y})=\alpha \mathrm{T}+\beta \mathrm{ISI}
\end{aligned}
$$

and ask: What ratio of coefficients $(\alpha / \beta)$ of $\mathrm{T}$ and of ISI gives the best predictions? This ratio may be called an on/off coefficient ratio because it expresses the relative value of continued exposure of the two stimuli vs a blank interval of equal duration.

The obvious technique for estimating the coefficients of $T$ and of ISI would seem to be multiple regression analysis. However, ordinary multiple regression analysis is ruled out because the relation of response accuracy (dependent variable) to $\mathrm{T}$ and ISI (independent variables) is highly nonlinear. For example, accuracies greater than $100 \%$ are impossible, and many conditions produced response accuracies in the range of $90 \%$ to $100 \%$. While the appropriate linear combination of $\mathrm{T}$ and of ISI may be expected to predict the order of response accuracy, it cannot be expected to predict it directly in the sense of an interval scale. Any linear prediction would predict accuracies greater than $100 \%$ for large values of the independent variables ( $T, I S I$ ). As the underlying dependent variable is the amount of information the $S$ has acquired from the stimulus (Sperling, 1970) and response accuracy is-in practice-only an ordinal measure of information, a technique is needed that takes this into account.

\section{Nonmetric Regression Analysis}

Fortunately, a powerful new technique of nonmetric regression analysis is available (Carroll, in press). In Carroll's method the dependent variable is monotonically transformed so as to make it a linear function of the independent variables. A computer-implemented iterative method finds the monotonic transformation $\left(\mathrm{M}^{-1}\right.$, Eq. 1) of the dependent variable and the linear combination of the independent variables that maximizes the correlation between the two.

Table 1 gives the on/off coefficient ratios and correlations computed by Carroll's method for the source paper's data. The high correlations (average correlation $=0.94$ ) between the predicted values and the data indicate that Carroll's method yields good monotonic transformations and good linear predictions. The resulting on/off coefficient ratios are very nearly equal to 1.2 on the average. ${ }^{6}$ This prediction obviously is closer to 1.0 than to 2.0 . In fact, for the data selected in the source paper for special consideration (average of results from Conditions $A, D, E)$ the estimated on/off coefficient ratio is 1.01 $(r=0.98)$. This means that $T$ and ISI contribute equally to accuracy as predicted by the persistence theory and not in a ratio of 2 to 1 , as predicted by the source paper's total stimulus duration hypothesis. That 9 of the 10 on/off coefficient ratios are less than 2.0 (in fact, less than 1.65) would exclude the total stimulus duration hypothesis, even by a simple sign test.

\section{Quantal Hypotheses}

Because of its nonmetric nature, Carroll's method is not biased for or against any specific nonlinear hypothesis (e.g., a quantal hypothesis) relative to any other nonlinear (or even a purely linear) hypothesis. The resulting on/off coefficient ratios exclude the variable $2 \mathrm{~T}+$ ISI from contention, and hence they exclude the source paper's particular discontinuity hypothesis. But perhaps there is a quantal discontinuity in the variable $\mathrm{x}=\alpha \mathrm{T}+\beta \mathrm{ISI}$ where $\alpha / \beta$ is the optimal on/off coefficient ratio. This would be revealed by * a discontinuity in the monotonic transformation $M$. We ask, does the monotonic relation between the independent variable $x$ and the monotonically transformed independent variable $y=M(x)$ show a discontinuity at some critical quantal time period, $\mathrm{x}_{\mathrm{q}}$ ? Without a specific quantal hypothesis, statistical testing is difficult, but to visual inspection the $y$ vs $x$ graphs simply do not suggest the presence of any quantal discontinuities. The analysis does not offer any support even for a post hoc quantal period.

In retrospect, the reason for the "large and apparently discontinuous break" in
Table 1

Estimated On/Off Coefficient Ratios for the Data from Greenberg et al's Five Kinds of Stimuli and Two Subjects. The number of random letters $(\ell)$ contained in each of the two stimuli is indicated in parenthesis. Correlations of the predicted scores with the monotonically transformed observed scores

\begin{tabular}{|c|c|c|c|}
\hline $\begin{array}{l}\text { Proce- } \\
\text { dure }\end{array}$ & Subject & $\begin{array}{c}\text { On/Off } \\
\text { Coefficient } \\
\text { Ratio }\end{array}$ & Correlation \\
\hline$A(1 \ell)$ & $\begin{array}{l}1 \\
2\end{array}$ & $\begin{array}{l}0.607 \\
0.897\end{array}$ & $\begin{array}{l}0.9266 \\
0.9565\end{array}$ \\
\hline$B(3 \ell)$ & $\begin{array}{l}1 \\
2\end{array}$ & $\begin{array}{l}1.005 \\
1.119\end{array}$ & $\begin{array}{l}0.9632 \\
0.8896\end{array}$ \\
\hline$C(5 l)$ & $\begin{array}{l}1 \\
2\end{array}$ & $\begin{array}{l}1.644 \\
2.107\end{array}$ & $\begin{array}{l}0.9486 \\
0.9727\end{array}$ \\
\hline $\mathrm{D}\left(3 \ell_{w}\right)$ & $\begin{array}{l}1 \\
2\end{array}$ & $\begin{array}{l}1.167 \\
1.289\end{array}$ & $\begin{array}{l}0.9771 \\
0.9193\end{array}$ \\
\hline$E(5 \ell w)$ & $\begin{array}{l}1 \\
2\end{array}$ & $\begin{array}{l}1.614 \\
1.487\end{array}$ & $\begin{array}{l}0.9007 \\
0.9342\end{array}$ \\
\hline \multicolumn{2}{|c|}{$\begin{array}{l}\text { Overail }(A, D, E) \\
\text { Overall }(A, B, C, D, E)\end{array}$} & $\begin{array}{l}1.012 \\
1.243\end{array}$ & $\begin{array}{l}0.9840 \\
0.9836\end{array}$ \\
\hline
\end{tabular}
are given at the far right.

response accuracy (of Conditions A, D, E) as a function of $2 \mathrm{~T}+$ ISI can be seen by considering, instead, accuracy in terms of $\mathrm{T}+\mathrm{ISI}$. The values of T + ISI fall into two ranges: 6 to $35 \mathrm{msec}$ (accuracy range $15.5 \%-56.8 \%)$ and 50 to $175 \mathrm{msec}$ (accuracy range $81.3 \%-99.0 \%$ ). (In fact, the response-range boundaries are defined by the same conditions as the stimulus-range boundaries.) The missing range of response accuracies between $56.8 \%$ and $81.3 \%$ (the "discontinuous break") appears to correspond exactly to a missing range of stimuli, stimuli with $\mathbf{T}+$ ISI between 35 and $50 \mathrm{msec}$.

To test the range-correspondence conjecture, we note that there is approximate proportionality between $\mathrm{T}+\mathrm{ISI}$ and response accuracy in the lower range, so it is appropriate to characterize the sizes of the missing ranges of stimuli and of responses by the ratio of their largest to their smallest member. The ratio of the upper to lower bounds of stimuli is $50 \mathrm{msec} / 35 \mathrm{msec}=1.43$ and the corresponding response ratio is $81.3 \% / 56.8 \%=1.43$. The estimates of the sizes of the two ranges agree quite well, indicating that the missing range of response accuracies (the "discontinuous break") is quantitatively predictable from the missing range of stimulus conditions.

\section{SECOND-ORDER PREDICTION}

Although the on/off coefficient ratios are near 1.0 , most values are greater than 1.0. Four factors that would affect the on/off coefficient ratio are considered now.

(1) When luminance is held constant and duration is diminished, the total energy in the flash is diminished; if the exposure is extremely brief, then the fiash will be 
invisible. Thus, for extremely brief exposures, exposure duration becomes more critical than interstimulus interval, and the on/off coefficient ratio would be greater than 1.0 to reflect this fact.

(2) Haber and Standing (1969) observed recently that persistence of stimulus visibility after stimulus turn-off is relatively independent of exposure duration. When the exposure duration of the second stimulus is appreciable (e.g., $50 \mathrm{msec}$ ) relative to visual persistence (e.g., $200 \mathrm{msec}$ ), then exposure duration may contribute significantly to the total time available for processing the second flash and thus to accuracy of report. Again, this effect of exposure duration on visibility of the second stimulus is not matched by an equal effect of a blank interval, and it would tend to increase the magnitude of the on/off coefficient ratio.

(3) In the source paper's oscilloscope displays, letters are composed of only a few points. Occasionally, a substantial fraction of the points will be common to the first and second stimulus and thus remain unchanged during the entire presentation. At 1-msec ISIs, therefore, Ss may experience difficulty in deciding that a new display has occurred. The difficulty seems to be most serious when oniy one letter is presented, perhaps because it offers the least opportunity for observing a change. With one-letter stimuli (Condition A), the on/off coefficient ratios are less than 1.0 for both Ss. This means that introducing a blank interval actually improved performance more than did an equivalent increase in the exposure duration of each stimulus.

(4) The four largest on/off coefficient ratios in Table 1 occur for five-letter stimuli: random letters (C) and words (E). These stimuli contain the most letters of any in the experiment; the additional processing time of the second stimulus that increased exposure duration could provide would account partially for the large coefficients in these procedures.

Another consequence of the persistence hypothesis is that reports of the first stimulus usually will be less accurate than reports of the second because processing of the first stimulus is interrupted by the onset of the second. Exceptions may occur when more letters are presented than can later be recalled and when ample time is available to process the first stimulus. The $S$ has an option of filling up his memory with letters from the first stimulus and having little space left over for letters from the second. However, only a few of the source paper's 140 presentation conditions satisfy these prerequisites. The relevant data pro inted therein are restricted to averaged data, and these are given only for one $S$. This $S$ reported the second stimulus more acuately on the average than the first for al! five kinds of stimuli, a result that is consistent with the persistence hypothesis.

\section{CONCLUSIONS}

In vicu of the possible artifacts that would lind to increase the value of the on/off cosfficient ratio, it is all the more remarkable that the analysis yields ratios so near to 1.0 , i.e., such great equality for the effects of continued exposure and the effects of a blank interval. The conclusions about the source paper's data from this are: (1) processing cut-off (of the first stimulus) by the onset of the second stimulus was the main factor to limit performance, (2) visibility per se at brief exposure durations (as opposed to processing cut-off) only rarely was the limiting factor in performance, and (3) visual persistence is sufficiently long so that the limited range of exposure durations did not affect performance much. In particular, memory, not processing cut-off, usually was the factor limiting responses to the second stimulus. All in all, the results of the analysis are consistent with the persistence-andcontinuous-processing theory and exclude the hypotheses of the source paper.

\section{REIERENCES}

CARROLL, J. D. Individual differences and multidimensional scaling. In R. N. Shepard, A. K. Romney, S. Nerlove (Jids.), Multidimensional scaling. Theory and application in the behavioral sciences. In press. GRFENBFRG, M., HELIER, M. S., \& MAYZNER, M. S. Information processing of letter and word pairs as a function of on and off times. Perception \& Psychophysics, 1968 , 4, 357-360.

HABER, R. N., \& STANDING, L. G. Direct measures of short-term visual storage. Quarterly Journal of Experimental Psychology, 1969, 21, 43-56.

SPERLING, G. A model for visual memory tasks. Human liactors, 1963, 5-31.

SPERLING, G. Successive approximations to a model for short-term memory. Acta Psychologica, 1967, 27, 285-292.

SPERLING, G. Information retrieval from two rapidly consecutive stimuli. Internal memorandum, Bell Telephone Laboratories, 1969.

SPERLING, G. Short-term memory, long-term memory, and scanning in the processing of visual information. In F. A. Young and D. B. Lindsley (Eds.), The influence of early experience on visual information processing. Washington, D.C: National Academy of Sciences, 1970. (In press.)

\section{NOTES}

1. Two aspects of the procedure make interpretation of the results difficult. In the case of words, the $S s$ are exposed to the entire set of 120 words about a dozen times. This means that in the second phase of the experiment, Ss often must be able to identify a word correctly even when they see only a single letter. The data tend to confirm this hypothesis-a higher percentage of letters is reported correctly from the three- or five-letter words than from the one-letter stimuli. Nevertheless, data from both phases are pooled. The other problem is that separate scores are not given for responses to the first stimulus and to the second stimulus.

2. "... while a gradual increase in percent correct detections occurs up to about $50 \mathrm{msec}$ of total processing time, a marked discontinuity appears to occur approximately between 50 and $60 \mathrm{msec}$ of total processing time, with per cent correct detections jumping abruptly from about $40 \%$ correct detections to about $90 \%$ correct detections over a total processing time span of only 10 msec [Abstract, p. 357]."

"The most striking result, of this analysis would appear to be the generally linear and gradual increase, with one exception, in per cent correct detections up to approximately $51 \mathrm{~m} \mathrm{sec}$, followed by a very large and what appears to be a discontinuous break in the per cent correct detections between 51 and $60 \mathrm{msec}$ [p. 359]."

3. See, for example, Note 2.

4. P. 359. See also Note 2.

5. There are certain restrictions to Principle 2. For example, time-resolution does not exceed about $10 \mathrm{msec}$ in the light-adapted eye so that events occurring within $10 \mathrm{msec}$ usually can be considered to be simultaneous; interactions between successive stimuli occur mainly in regions that contain boundaries from both. However, for the purpose of analyzing the source paper's data, these restrictions can be neglected.

6 . The average of a ratio of the two coefficients is an inappropriate biased measure of central tendency. It depends, for example, on the way in which the coefficients are taken, i.e., $C_{1} / C_{2}$ or $C_{2} / C_{1}$. There are several appropriate measures. The median is one. A more powerful measure of central tendency is obtained by computing the coefficient-ratio from a pool of all the data. linally, an appropriate transformation for dealing with ratios is the $\tan ^{-1}$ transformation, which treats the coefficient-ratio $y$ as the tangent of a vector. The transformed average then is $\tan$ [Ave $\left(\tan ^{-1} y\right)$ ]. In fact, measures of central tendency computed by all three incthods [median, overall, $\tan \left(\tan ^{-1}\right)$ ] agree to within $2 \%$; the overall measure is given in Table 1.

7. Note added in press: Since preparation of this article, R. N. Haber and Linda S. Nathanson (Perception \& Psychophysics, 1969, 5, 359-361) have published an elegant experimental study of the "processing of sequentially presented letters" that comes to the same conclusion as the present analysis, namely: "It was found that the processing time (onset to onset) predicted the number of letters correctly reported, regardless of the partition between on time and off time [p. 359]."

(Accepted for publication May 24, 1970.) 